\title{
Fetal Nasal Bone Length in the Period of 11 and 15 Weeks of Pregnancy in the Filipino Population
}

\author{
Leodoro J. Labrague ${ }^{1, *}$, Lynette C. Tan ${ }^{2}$ \\ ${ }^{1}$ Samar State University, Philippines \\ ${ }^{2}$ University of Santo Tomas, Manila, Philippines \\ *Corresponding author: Leo7_ci@yahoo.com
}

Received February 25, 2013; Revised December 17, 2013; Accepted December 23, 2013

\begin{abstract}
In the recent years, sonographic images of fetal nose have gained popularity in detecting trisomy 21 and other aneuploidies. Reference values for nasal bone length have been reported in different countries and ethnic populations. However, there was no reference range for the fetal nasal bone length (NBL) in the Filipino population. This prospective, cross-sectional study was conducted to establish reference values for the nasal bone length (NBL) at $11-15$ weeks of gestation in the Filipino population. Ultrasonographic visualization of 74 fetuses at 11th to 15th week gestation was carried out during the months of August 2010 to January 2011. Fetal nasal bone was measured through sagittal section of the fetal profile. Fetal nasal bone length measurement was successfully performed in all 74 cases. The mean nasal bone lengths were $1.97 \mathrm{~mm}, 2.37 \mathrm{~mm}, 2.90 \mathrm{~mm}, 3.49 \mathrm{~mm}$, and $4.05 \mathrm{~mm}$ between 11th, 12th, 13th, 14th, and 15th gestational weeks respectively. Nasal bone length increased linearly with advancing gestational age (GA) and crown rump length (CRL). Despite of the limitations posed by this investigation, reference values for the nasal bone length (NBL) at $11-15$ weeks of gestation in the Filipino population was identified.
\end{abstract}

Keywords: fetal nasal bone, ultrasound, trisomy 21, Down syndrome, First-trimester screening

Cite This Article: Leodoro J. Labrague, and Lynette C. Tan, "Fetal Nasal Bone Length in the Period of 11 and 15 Weeks of Pregnancy in the Filipino Population." American Journal of Medical Sciences and Medicine 1, no. 6 (2013): 110-113. doi: 10.12691/ajmsm-1-6-3.

\section{Introduction}

Screening for chromosomal abnormalities during the 1980s was initially based only on the pregnant woman's age. In the later years, it has been broadened and included maternal serum alpha-fetoprotein, [1] maternal serum biochemical markers [2], and nuchal translucency thickness measurements. [3] Recently, sonographic images of the fetal nose during the second trimester have gained popularity in accurately predicting the risk of the chromosomal birth defect such as Down syndrome. [4,5].

The fetal nasal bone can be visualized by sonography at 11 to $13+6$ weeks of gestation in $99.5 \%$ of chromosomally normal fetuses [4]. However, in about $60-70 \%$ of fetuses with trisomy 21 and in about $2 \%$ of chromosomally normal fetuses the nasal bone is not visible at 11-13 weeks' gestation due to delayed ossification or hypoplasia. [4] Few other studies reported that absence of nasal bone by first trimester ultrasound was significantly associated with Down syndrome. Otano et al [6] reported absence of fetal nasal bone in $60 \%$ of fetuses with Down syndrome, while Orlandi et al. [7] observed absence of fetal nasal bone in $67 \%$ of fetuses with trisomy 21 and $1 \%$ in chromosomally normal fetuses. Prefumo et al [13] also showed a trend of a higher incidence of an absent nasal bone in fetuses of healthy Asian mothers. Moreover, hypoplasia and absence of the nasal bone were also reported in $36 \%$ and $2.5 \%$ respectively in aborted fetuses.
[8] Furthermore, if combined with other screening methods such as maternal serum free beta-human chorionic gonadotropin (B-hCG), maternal age, fetal nuchal translucency (NT) thickness, and pregnancyassociated plasma protein (PAPP-A) at $11-14$ weeks, it is projected to increase detection rate to $85 \%$ with a falsepositive rate of $5 \%$ [4].

It is essential to note that nasal bone length measurement is impacted by race and ethnicity. Therefore, racial adjustment is needed in the measurement of fetal nasal bone in screening for trisomy 21 during pregnancy [15].

Reference values for nasal bone length have been reported in different countries and ethnic populations $[9,10,11,12,14,18]$. However, to the authors' knowledge, no study ascertaining reference ranges for the nasal bone length in a Filipino population have been carried out. Thus, this prospective, cross-sectional study was conducted to establish reference values for the nasal bone length (NBL) at $11-15$ weeks of gestation in the Filipino population.

\subsection{Research Objective}

This study was conducted to establish reference values for the nasal bone length (NBL) at 11 - 15 weeks of gestation in the Filipino population.

\section{Methodology}


This prospective, cross-sectional study was conducted in the clinical division of the department of Obstetrics and Gynecology at the University of Santo Tomas Hospital, Philippines during the months of August 2010 to January 2011.

The study sample included 74 pregnant women who had been submitted for first trimester ultrasound scanning. For the purpose of this study, inclusion criteria were set for the purpose of delimitation such as; (a) women with singleton pregnancy, (b) gestational age of 11 to 14 based on the last menstrual period and as confirmed in the ultrasound, and (c) absence of malformations or pregnancy complications. The study protocol was approved by the Ethics Committee of the University of Santo Tomas Hospital.

In measuring the nasal bone length, a sagittal section of the fetal profile was obtained with the ultrasound transducer at an angle between 45 to 135 to the facial plane. The image was magnified so that the fetal head and upper thorax were present on the $75 \%$ of the screen. The nasal bone and nasofrontalsynostosis, which appear as an anechoic area on the glabellar region, were identified besides other two liner parallel and echogenic images corresponding to the skin interface right above the nasal bone. Once the appropriate plane was identified, measurement of the nasal bone was performed. The correct plane for the measurement of the fetal nasal bone length is illustrated in Figure 1.

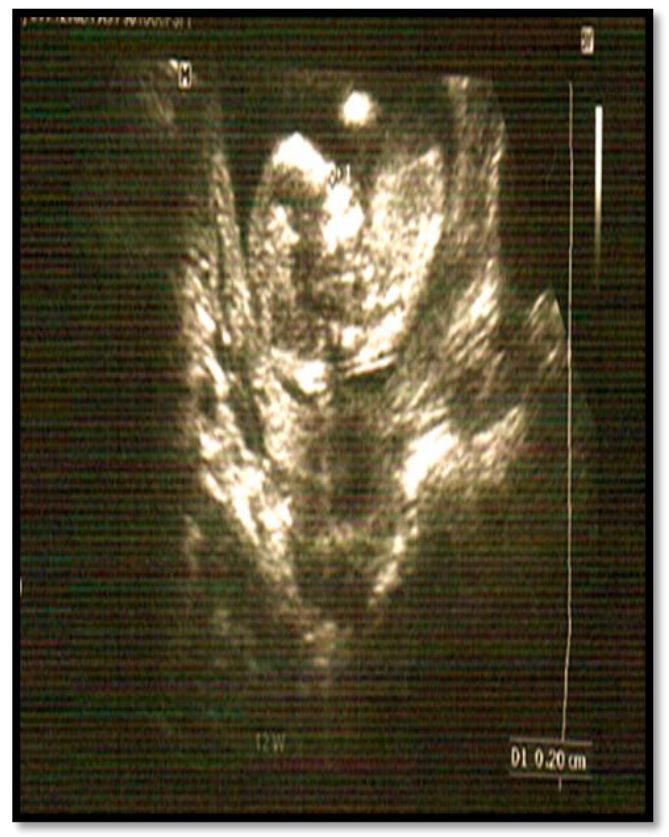

Figure 1. Measurement of the fetal nasal bone length
All the sonographic examinations were performed in GE Voluson 750 equipment coupled with a convex transducer $(3.5-5.0 \mathrm{MHz})$ and the trans-abdominal approach was adopted for all the measurements.

Following data collection, statistical analysis was performed using Microsoft Excel and were computed by SPSS version 19. Scatter plots for nasal bone length as a function of gestational age and crown rump length were constructed. The $5^{\text {th }}$, and $95^{\text {th }}$ percentile values were calculated for each gestational week. Logistic Regression analysis was done to identify correlations between nasal bone length, gestational age, and crown rump length.

\section{Results}

Fetal nasal bone length measurement was successfully performed in all 74 cases. The fetal nasal bone was visualized and measured in $100 \%$ of the fetuses. The average maternal age was 28.5 years with standard deviation of 5 years. The mean nasal bone length was 2.64 $\mathrm{mm}$ with an overall SD of $0.86 \mathrm{~mm}$.

Table 1 shows the average means, standard deviations, and reference measurements in the 2.5\%, 5\%, 50\%, 95\%, and $97.5 \%$ percentiles for fetal nasal bone length during the period of 11th to 15th weeks of gestational age. As observed, the nasal bone lengths were 1.97, 2.37, 2.90, 3.49 , and 4.05 between 11th, 12th, 13th, 14th, and 15th gestational weeks respectively. Table 2 presents nasal bone length in the first trimester in different ethnic groups.

Figure 2 shows the linear relationship between NBL and GA. The nasal bone length increased significantly with gestational age $(\mathrm{P}=.01)$. The nasal bone length increased linearly with advancing gestational age and was described by the following equation: $y=0.518 x-3.776$ $(\mathrm{R} 2=0.738)$. When the regression analyzes of relationship between nasal bone and CRL was done, regression equation was found as: $\mathrm{y}=18.50 \mathrm{x}+13.14$ ( $\mathrm{R} 2$ $=0.811$ ) (Figure 3). Also, statistically a significant correlation was found between gestational age and CRL (Figure 4).

Table 1. Nasal Bone Length in the First Trimester

\begin{tabular}{|c|c|c|c|}
\hline \multirow{2}{*}{ Gestational Age } & \multirow{2}{*}{$\begin{array}{c}\text { Number of } \\
\text { Cases }\end{array}$} & \multicolumn{2}{|c|}{ Nasal Bone Length } \\
\cline { 3 - 4 } & 27 & Mean & SD \\
\hline 11 & 20 & 1.97 & 0.25 \\
\hline 12 & 9 & 2.37 & 0.55 \\
\hline 13 & 8 & 2.90 & 0.54 \\
\hline 14 & 10 & 3.49 & 0.61 \\
\hline 15 & & 4.05 & 0.38 \\
\hline
\end{tabular}

Table 2. Nasal Bone Length in the First Trimester in Different Ethnic Groups (5\% and 95\% percentile)

\begin{tabular}{|c|c|c|c|c|c|c|c|c|c|c|}
\hline \multirow{2}{*}{ Age of Gestation } & \multicolumn{2}{|c|}{ This study } & \multicolumn{2}{|c|}{$\begin{array}{c}\text { Brazilian } \\
\text { Cossi (2008) } \\
\end{array}$} & \multicolumn{2}{|c|}{$\begin{array}{c}\text { Korean } \\
\text { Moon (2006) }\end{array}$} & \multicolumn{2}{|c|}{$\begin{array}{c}\text { Thai } \\
\text { Suwanrath (2013) }\end{array}$} & \multicolumn{2}{|c|}{$\begin{array}{c}\text { Turkish } \\
\text { Yayla (2011) } \\
\end{array}$} \\
\hline & $5^{\text {th }}$ & $95^{\text {th }}$ & $5^{\text {th }}$ & $95^{\text {th }}$ & $5^{\text {th }}$ & $95^{\text {th }}$ & $5^{\text {th }}$ & $95^{\text {th }}$ & $5^{\text {th }}$ & $95^{\text {th }}$ \\
\hline 11 & 1.7 & 2.5 & 1.1 & 2.0 & 1.2 & 1.9 & 1.1 & 1.9 & 1.3 & 2.1 \\
\hline 12 & 1.6 & 3.5 & 1.4 & 2.5 & 1.4 & 2.1 & 1.1 & 2.5 & 1.5 & 2.4 \\
\hline 13 & 2.1 & 3.9 & 1.7 & 3.0 & 1.6 & 2.3 & 1.5 & 2.6 & 1.8 & 2.8 \\
\hline 14 & 2.9 & 4.8 & 1.8 & 3.5 & 1.7 & 2.6 & ND & ND & ND & ND \\
\hline 15 & 3.7 & 4.9 & ND & ND & ND & ND & ND & ND & ND & ND \\
\hline
\end{tabular}




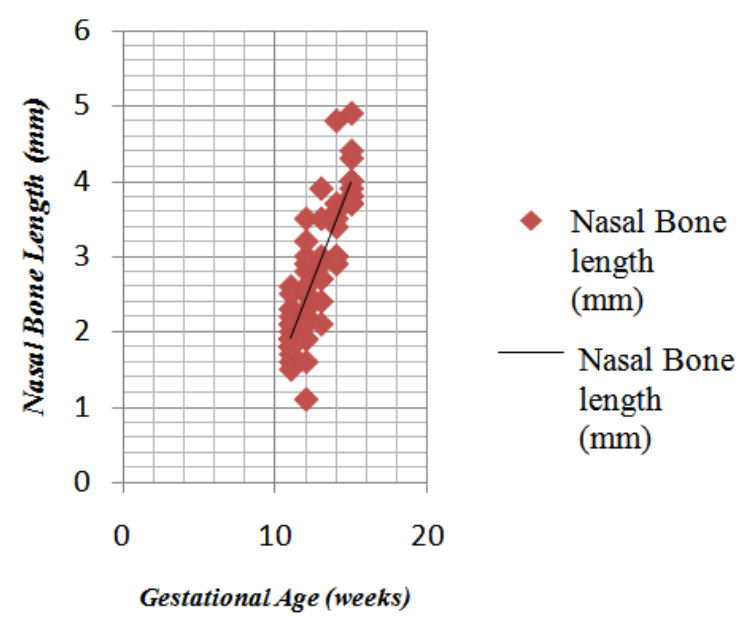

Figure 2. Scatter plot between nasal bone length and gestational age

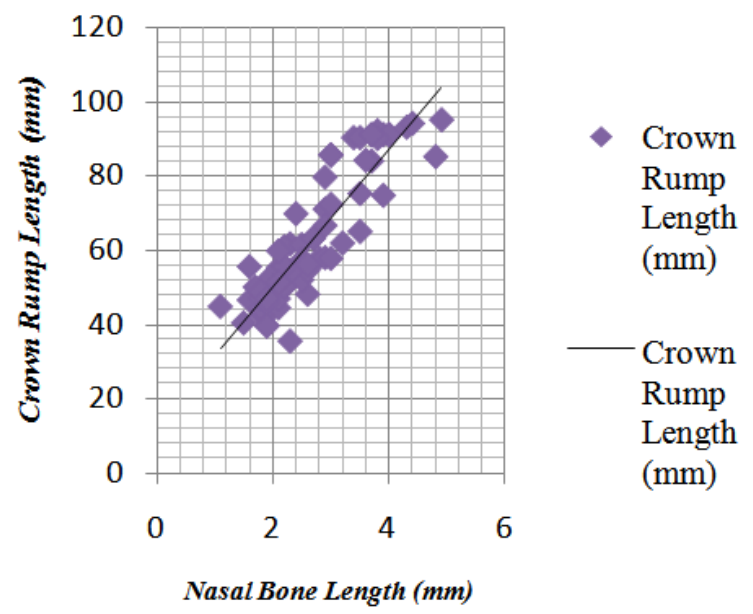

Figure 3. Scatter plot between nasal bone length and crown rump length

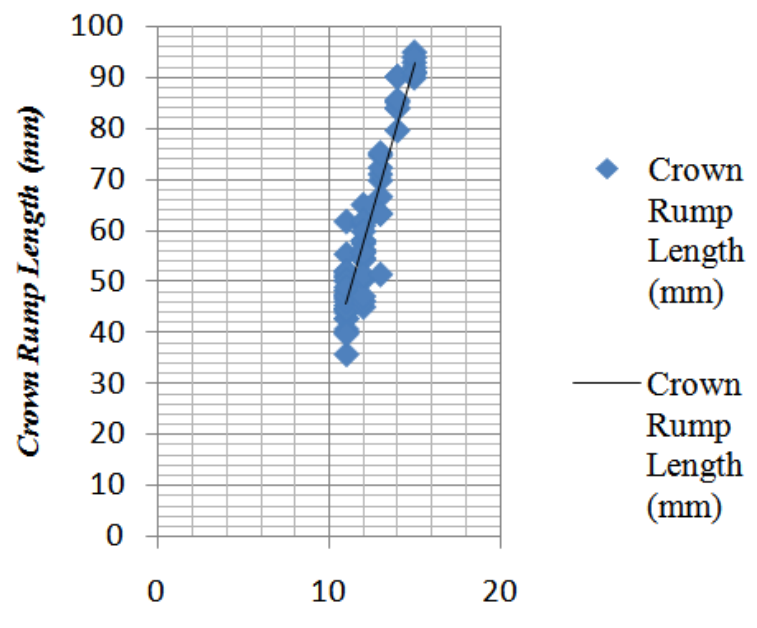

Gestational Age (weeks)

Figure 4. Scatter plot between gestational age and crown rump length

\section{Discussions}

This prospective, cross-sectional study was established reference values for the nasal bone length (NBL) at 11 15 weeks of gestation in the Filipino population. Reference values for nasal bone length have been reported in different countries and ethnic populations. In the current study, the mean nasal bone length ranged from $1.97 \mathrm{~mm}$ to $4.05 \mathrm{~mm}$. When compared with other ethnic groups [9,10,12,14], nasal bone length in Filipino population appears to be longer. For instance, in the Korean population, the average nasal bone length were 1.5 $\mathrm{mm}$ and $2.1 \mathrm{~mm}$ for 11 and 14 weeks gestation [9] The NBLs at the 50th percentile in Latin American population were $1.5,1.7$, and $1.9 \mathrm{~mm}$ at 11,12 , and 13 weeks of gestation, respectively. [10] Meanwhile, the nasal bone length in Thai fetuses at 11 to 15 weeks were $1.4 \mathrm{~mm}$ (range, 1.1-1.9), $1.7 \mathrm{~mm}$ (range, 1.1-2.5), and $2.1 \mathrm{~mm}$ (range, 1.5-2.6) at gestational age of 11,12 , and 13 weeks respectively [18]. In the Turkish population, the median values of nasal bone lengths were $1.7 \mathrm{~mm}, 1.9 \mathrm{~mm}$, and $2.2 \mathrm{~mm}$ for 11,12 and 13 gestational weeks respectively. [12] In the study developed by Cossi et al [14] in a Brazilian population, the nasal bone length in all cases ranged between $1.69 \mathrm{~mm}$ and $2.94 \mathrm{~mm}$ at 11th and 15th weeks. It can be gleaned further that the average values for nasal bone measurements vary from one study to another. In our study, the mean NBL during the 11th to 15 th weeks were relatively higher compared to previous studies undertaken $[9,12,14,18]$. It is essential to note therefore, based on the previous findings that race and ethnicity have an impact on fetal nasal bone length. In agreement with previous authors [15], maternal ethnicity should be required for utilizing the fetal nasal bone length in the screening for trisomy 21 at the first trimester of gestation. However, other factors such as observer variability and quality of machine used should not be undermined.

It was also evident that the nasal bone length in all cases was increased linearly as pregnancy advances. This result is in keeping with the result obtained by Ceciro et al [4] Cossi et al [14], Moon et al [9], Yayla et al [12], Chen et al. [16], and Cusick et al [17]. Nasal bone length was also highly correlated with CRL. It can be concluded based on this result that as pregnancy progresses, measurements of nasal bone length becomes easier and feasible.

While these data are important as it is the first analysis conducted in the country, nevertheless it has some limitations. First, this investigation was conducted among pregnant women from one province only, thus exclusion of pregnant women from other provinces may limit the generalizability of this investigation. Secondly, the small sample size might have affected the statistical power and effect size of the study. Thus, future studies utilizing bigger samples may be conducted to increase statistical power and effect size.

\section{Conclusion}

Despite of the limitations posed by this investigation, our findings provided reference values for the nasal bone length (NBL) at 11 - 15 weeks of gestation in the Filipino population. Findings also revealed that nasal bone length in the samples vary from other race and ethnic group. In agreement with previous authors, examination of fetal nasal bone at 11 - 15 weeks could have major implications in screening for trisomy 21 by maternal age and fetal nuchal translucency. The findings added 
knowledge to the existing body of literature on research related to trisomy 21 screening during pregnancy.

\section{Competing Interest}

The author(s) declare that they have no competing interests.

\section{References}

[1] Cuckle HS, Wald NJ, Lindenbaum RH. Maternal serum alphafetoprotein measurement: a screening test for Down syndrome. Lancet 1984; 1: 926-929.

[2] Wald NJ, Cuckle HS, Densem JW, et al. Maternal serum screening for Down's syndrome in early pregnancy. BMJ 1988; 297: 883-887.

[3] Snijders, R. J., Noble, P., Sebire, N., Souka, A., \&Nicolaides, K. $\mathrm{H}$. UK multicentre project on assessment of risk of trisomy 21 by maternal age and fetal nuchal-translucency thickness at 10-14 weeks of gestation. Fetal Medicine Foundation First Trimester Screening Group. Lancet 1998; 352(9125), 343.

[4] Cicero, S., Curcio, P., Papageorghiou, A., Sonek, J., \&Nicolaides, $\mathrm{K}$. Absence of nasal bone in fetuses with trisomy 21 at 11-14 weeks of gestation: an observational study. The lancet 2001; 358 (9294), 1665-1667.

[5] Labrague, L.J. Expectant management in dichorionicdiamnionic twin pregnancy discordant for anencephaly. Standard Scientific Research and Essays 2013; 1(8):164-167.

[6] Otaño L, Aiello H, Igarzábal L, Matayoshi T, Gadow EC. Association between first trimester absence of fetal nasal bone on ultrasound and Down syndrome. PrenatDiagn 2002; 22:10, 930-2.

[7] Orlandi, F., Bilardo, C. M., Campogrande, M., Krantz, D. Hallahan, T., Rossi, C., \&Viora, E. Measurement of nasal bone length at 11-14 weeks of pregnancy and its potential role in Down syndrome risk assessment. Ultrasound in obstetrics \& gynecology 2003; 22:1, 36-39.
[8] Keeling, J. W., Hansen, B. F., \&Kjaer, I. Pattern of malformations in the axial skeleton in human trisomy 21 fetuses. American journal of medical genetics 1998; 68:4, 466-471.

[9] Moon, M. H., Cho, J. Y., Lee, Y. M., Lee, Y. H., Yang, J. H., Kim, M. Y., \& Park, S. H.Nasal bone length at 11-14 weeks of pregnancy in the Korean population. Prenatal diagnosis 2006; 26:6, 524-527.

[10] Casasbuenas, A., Wong, A. E., \& Sepulveda, W. First-trimester nasal bone length in a normal Latin American population. Prenatal diagnosis 2009; 29:2, 108-112.

[11] Sutthibenjakul, S., Suntharasaj, T., Suwanrath, C., Kor-anantakul, O., \&Geater, A. A Thai reference for normal fetal nasal bone length at 15 to 23 weeks' gestation. Journal of Ultrasound in Medicine 2009; 28:1, 49-53.

[12] Yayla, M., Ergin, R. N., \&Göynümer, G. Normative values of fetal nasal bone lengths of Turkish singleton pregnancies in the first trimester. Journal of the Turkish-German Gynecological Association 2011; 12.

[13] Prefumo F, Sairam S, Bhide A, Penna L, Hollis B, Thilaganathan B. Maternal ethnic origin and fetal nasal bone at 11-14 weeks of gestation. BJOG 2004; 111:109-112.

[14] Cossi, P.S, Junior, E.A., Bussamra, L.C., Filho, H.A., Nardazzo, L.M., Moron, A.F. Measurement of fetal nasal bone length in the period between 11 and 15 gestational weeks in a Brazilian population: a preliminary study. Radiol Bras 2008; 41:3, 155-158.

[15] Collado, F., Bombard, A, Li, V., et al. Ethnic variation of fetal nasal bone length between $11-14$ weeks' gestation. PrenatDiagn 2006; 26, 703-7

[16] Chen M, Lee CP, Tang MH. First trimester examination of fetal nasal bone in the Chinese population. PrenatDiagn 2006;26, 524 7.

[17] Cusick, MD, Provenzano J, Sullivan CA, Gallousis FM, Rodis JF Fetal nasal bone length in Euploid and Aneuploid fetuses between 11 and 20 weeks gestation. J ultrasound Med. 2004; 23:1327 1333

[18] Suwanrath et al.: Reliability of fetal nasal bone length measurement at 11-14 weeks of gestation. BMC Pregnancy and Childbirth 2013 13:7. 